\title{
MS05-02 | Structural Insights Into Signal Processing to Chromatin
}

Panne, Daniel (University of Leicester, Leicester, GBR)

Signals that emerge from cellular receptors ultimately impinge on chromatin to regulated gene expression. As part of such signalling reactions chromatin becomes extensively modified. The precise mechanisms that allow cellular signalling to control chromatin modification and how such modifications contribute to gene regulation remain unclear. One such modification is Lysine acetylation which has long been correlated with active gene transcription. p300 is a multivalent adaptor for Transcription factors (TFs) and an acetyltransferase that is typically recruited to enhancers and contributes to gene regulation by acetylating chromatin and other transcriptional regulators. We have recently discovered that the activation of p300 depends on the activation and oligomerization status of TFs. We found that TF dimerization enables trans-autoacetylation of p300 in a highly conserved and intrinsically disordered autoinhibitory lysine-rich loop. Structural and biochemical studies, in combination with molecular modelling, indicate that TF dimerization enables trans-autoacetylation of the lysine-rich loop thus relieving autoinhibition and enabling activation of the enzyme. A large fraction of TFs operate as functional dimers and dimerization is frequently inducible by exogenous stimuli. We therefore propose that cellular signalling directly controls activation and genome targeting of p300 thus explaining why chromatin acetylation is associated with transcriptionally active chromatin. Our working model is that acetylation contributes to nucleation and concentration of dynamic TF-p300 assemblies on enhancers and thereby to active gene regulation. Dysregulation of p300-mediated chromatin acetylation by oncogenic TFs can lead to formation of aberrant chromatin condensates and profound changes in gene expression programs that drive the malignant cell state. 